\title{
Correspondence
}

\section{Post-Intubation Empirical Fluid Resuscitation as an Early Step to Prevent Acute Kidney Injury in Mechanically Ventilated COVID-19 Patient}

\author{
Amarjeet Kumar ${ }^{1}$, Ajeet Kumar2, ${ }^{2 *}$, Chandni Sinha ${ }^{2}$, Prabhat Kumar Singh ${ }^{3}$, Chethan Vamshi², Gayatri D. Sagdeo ${ }^{2}$ \\ ${ }^{1}$ Department of Trauma and Emergency, All India Institute of Medical Sciences, Room no 505, B-Block, OT Complex, Patna, India \\ ${ }^{2}$ Department of Anaesthesiology, All India Institute of Medical Sciences, Room no 502, B-Block, OT Complex, Patna, India \\ ${ }^{3}$ All India Institute of Medical Sciences, Patna, India
}

\section{ARTICLE INFO}

Article History

Received 21 May 2021

Accepted 11 October 2021

\section{Keywords}

Acute kidney injuries

COVID-19

fluid resuscitation

mechanical ventilation

\begin{abstract}
Acute Kidney Injury (AKI) is one of the major complication of the Coronavirus Disease 2019 (COVID-19) infection, commonly manifested during the second week of infection. The pathophysiology and mechanisms of AKI in patients with COVID-19 have not been fully elucidated and seem to be multifactorial. There is currently no common consensus regarding the optimal amount of fluid resuscitation specifically for severe acute respiratory syndrome coronavirus 2 (SARS-CoV-2)-infected patients in shock. There is paucity of knowledge regarding prevention of post-intubation hypotension and oliguria in COVID-19 patients. We suggest fluid resuscitation by $1 \mathrm{~L}$ of crystalloid immediately following endotracheal intubation of adult COVID ARDS patients to compensate vasodilation and improve renal perfusion. Conservative fluid strategies should be followed in patient having history of congestive heart failure, chronic kidney disease, adrenal insufficiency and in patient who have already developed AKI with oliguric phase.
\end{abstract}

() 2021 First Affiliated Hospital of Zhengzhou University. Publishing services by Atlantis Press International B.V. This is an open access article distributed under the CC BY-NC 4.0 license (http://creativecommons.org/licenses/by-nc/4.0/).
Acute Kidney Injury (AKI) is one of the major complication of the Coronavirus Disease 2019 (COVID-19) infection, commonly manifested during the second week of infection [1]. Clinical manifestation ranges from prerenal azotaemia to acute tubular necrosis. As per current evidence, the incidence of AKI is $>20 \%$ of hospitalized patients and $>50 \%$ of patients are admitted to Intensive Care Unit (ICU) [2]. The pathophysiology and mechanisms of AKI in patients with COVID-19 have not been fully elucidated and seem to be multifactorial. The development of AKI in COVID-19 patients depend upon patient age, severity of cytokine storm, the presence of sepsis, use of nephrotoxic drug, duration of Mechanical Ventilation (MV) and baseline serum creatinine level. Acute hypoxemic COVID-19 patients are also at a high risk of AKI as a complication of mechanical ventilation, particularly when treated with high Positive End-Expiratory Pressure (PEEP). High PEEP leads to increased intrathoracic pressure which result in increased renal venous pressure and reduced filtration [3]. Careful attention to volume status is needed to avoid hypovolaemia in these patients. There is currently no common consensus regarding the optimal amount of fluid resuscitation specifically for SARS-CoV-2-infected patients in shock. The evidence-based fluid resuscitation from the more general group of patients in shock, Acute Respiratory Distress Syndrome (ARDS), and other related conditions might have to be

"Corresponding author.Email: ajeetanaes@gmail.com

Peer review under responsibility of the First Affiliated Hospital of Zhengzhou University extrapolated in the COVID-19 patient population [4]. However, both inadequate and excessive fluid resuscitation could be harmful in these patients.

Oliguria following intubation is commonly seen in COVID 19 patients. In our clinical experience, patients who received additional bolus of fluid during or immediately after intubation had lesser incidence of oliguria than those patients who received no bolus.

Coronavirus disease 2019 patients are in a state of hypovolemia due to poor oral intake, insensible fluid loss due to fever, or diarrhoea that had preceded admission. This volume contraction is further aggravated by minimal fluid resuscitation, because of the concern about risk of rapid respiratory decompensation. High positive end-expiratory pressure strategies of COVID ARDS management may further reduce venous return. In addition, all forms of positive pressure mechanical ventilation can increase sympathetic tone, leading to secondary activation of the renin-angiotensin system [5]. Activation of renin-angiotensin system gives false sense of haemodynamic stability [normal mean arterial pressure (MAP)] in COVID-19 patients following intubation, despite of severely volume contracted state.

The pathophysiology of oliguria following intubation is poorly understood. Poor renal perfusion is the most possible cause of post-intubation oliguria in COVID-19. Factors responsible for post-intubation hypotension is vasodilatation due to relief from 
respiratory distress, use of sedatives and muscle relaxant during intubation, carbon dioxide washout effect, decrease in intraabdominal pressure because of rest to accessory muscle contraction and increase in intra-thoracic pressure due to high PEEP. Direct cause of AKI include direct viral cytotoxicity (because of angiotensinconverting enzyme 2-receptor for SARS-CoV-2, expressed in human kidney) [6].

Consensus statement of 25th conference of the acute disease quality initiative recommend patient stratification for risk of AKI based on their comorbidities (baseline CKD) and demographics [7]. The development and progress of AKI depend upon, severity of illness, process of care, along with the threshold for admission to the hospital and the ICU. Correlation between the initiation of MV and AKI is peculiar and its pathophysiologic mechanisms need to be fully understood. The association between MV and AKI in patients of COVID ARDS might be due to lung-kidney cross-talk [8]. Lung protective ventilation strategy may decrease the risk of AKI [9]. However, resistant hypoxia and in late stage hypercapnia create a major challenge for lung protective ventilation of COVID ARDS patients.

According to Surviving Sepsis COVID-19 guidelines 2020, for the acute resuscitation of adult COVID-19 shock patient, conservative fluid management is preferred over a liberal fluid strategy [10]. However, WHO COVID-19 guidelines suggest use of 250-500 mL crystalloid fluid as rapid bolus in first $15-30 \mathrm{~min}$ for the resuscitation of adult COVID-19 shock patient [11]. Pragmatic recommendations for the management of COVID-19 patients with shock in low- and middle-income countries by Maximous et al. [12] recommend use of crystalloids over colloids and balanced crystalloid over normal saline for the resuscitation of adult COVID-19 patient. They also suggest against the routine use of albumin for initial resuscitation of adults with COVID-19 and shock. For the resuscitation of COVID-19 patient, the International Fluid Academy [13] recommend bolus of $4 \mathrm{~mL} / \mathrm{kg}$ of balanced crystalloid over 10-15 min. Recommendations also include identification of causes of fluid deficit, assessment for presence of shock or hypoperfusion, fluid balance on admission in the hospital and on daily basis as cumulative fluid balance calculated, fluid responsiveness and progression of urea and electrolytes (at least once every $24 \mathrm{~h}$ of fluid prescription). Fluid responsiveness is assessed by functional haemodynamics [pulse pressure variation, passive leg raise test, end-expiratory occlusion test, or a combination]. However, as per UK Joint Anaesthetic and Intensive Care Guidelines [13] fluid overload should be avoided and more conservative administration may help improve respiratory function, which should be carefully balanced against the risk of inducing acute renal impairment. Care should be exercised in 'running patients too dry' in an effort to spare the lungs, as there are increased insensible fluid losses.

There is paucity of knowledge regarding prevention of postintubation hypotension and oliguria in COVID-19 patients. Flinspach et al. [14] in a monocentric observational study found that, there is unusual high requirement of sedatives in mechanically ventilated patients with COVID-19 compared to those available in the literature. In these patients we can extrapolate knowledge of perioperative fluid management in form of Compensatory Intravascular Volume Expansion (CVE) to counteract venodilation and cardiac depression from anesthesia as well as the hemodynamic effects of positive-pressure ventilation. CVE with 5-7 mL/kg of a balanced salt solution should occur prior to, or simultaneously with induction of general anesthesia provided there are no patient comorbidities prohibiting fluid administration [15]. So, we suggest fluid resuscitation by $5-8 \mathrm{~mL} / \mathrm{kg}$ of crystalloid immediately following endotracheal intubation of adult COVID ARDS patients to compensate vasodilation and improve renal perfusion. However, conservative fluid strategies should be followed in patient having history of congestive heart failure, chronic kidney disease, adrenal insufficiency and in patient who have already developed AKI with oliguric phase [16]. Renal Replacement Therapy should be reserved for patients who develop progressive azotemia, mixed metabolic and respiratory acidosis, increased diffuse interstitial and airspace opacities on a chest radiograph.

\section{CONFLICTS OF INTEREST}

The authors declare they have no conflicts of interest.

\section{AUTHORS' CONTRIBUTION}

All the authors contributed substantially to the work presented in this article. Amarjeet Kumar and Ajeet Kumar contributed to conceptualization and writing-original draft. Chandni Sinha contributed in writing-review and editing. Prabhat Kumar Singh contributed in supervision. Chethan Vamshi and Gayatri D. Sagdeo contributed to data curation and writing-review and editing.

\section{CONSENT OF PUBLICATION}

Written and informed consent for publication was taken from the parents.

\section{REFERENCES}

[1] Cheng Y, Luo R, Wang K, Zhang M, Wang Z, Dong L, et al. Kidney disease is associated with in-hospital death of patients with COVID-19. Kidney Int 2020;97;829-38.

[2] Hirsch JS, Ng JH, Ross DW, Sharma P, Shah HH, Barnett RL, et al. Acute kidney injury in patients hospitalized with COVID-19. Kidney Int 2020;98;209-18.

[3] Koyner, JL, Murray PT. Mechanical ventilation and lung-kidney interactions. Clin J Am Soc Nephrol 2008;3;562-70.

[4] Juneja D, Savio RD, Srinivasan S, Pandit RA, Ramasubban S, Reddy PK, et al. Basic critical care for management of COVID-19 patients: position paper of the Indian Society of Critical Care Medicine, Part II. Indian J Crit Care Med 2020;24;S254-S62.

[5] Dudoignon E, Moreno N, Deniau B, Coutrot M, Longer R, Amiot Q, et al. Activation of the renin-angiotensin-aldosterone system is associated with acute kidney injury in COVID-19. Anaesth Crit Care Pain Med 2020;39;453-5.

[6] Lely AT, Hamming I, van Goor H, Navis GJ. Renal ACE2 expression in human kidney disease. J Pathol 2004;204;587-93.

[7] Nadim MK, Forni LG, Mehta RL, Connor MJ, Liu KD, Ostermann $\mathrm{M}$, et al. COVID-19-associated acute kidney injury: consensus report of the 25th Acute Disease Quality Initiative (ADQI) Workgroup. Nat Rev Nephrol 2020;16;747-64. 
[8] Sang L, Chen S, Zheng X, Guan W, Zhang Z, Liang W, et al. The incidence, risk factors and prognosis of acute kidney injury in severe and critically ill patients with COVID-19 in mainland China: a retrospective study. BMC Pulm Med 2020;20;290.

[9] van den Akker JPC, Egal M, Groeneveld ABJ. Invasive mechanical ventilation as a risk factor for acute kidney injury in the critically ill: a systematic review and meta-analysis. Crit Care 2013; 17;R98.

[10] Alhazzani W, Møller MH, Arabi YM, Loeb M, Gong MN, Fan E, et al. Surviving sepsis campaign: guidelines on the management of critically ill adults with coronavirus disease 2019 (COVID-19). Intensive Care Med 2020;46;854-87.

[11] World Health Organization. Clinical management of severe acute respiratory infection (SARI) when COVID-19 disease is suspected. Available from: https://who.int/emergencies/diseases/ novel-coronavirus-2019/events-as-they-happen.

[12] Maximous S, Brotherton BJ, Achilleos A, Akrami KM, Barros LM, Cobb N, et al. Pragmatic recommendations for the management of COVID-19 patients with shock in low- and middle-income countries. Am J Trop Med Hyg 2021;104;72-86.

[13] Malbrain MLNG, Ho S, Wong A. Thoughts on COVID-19 from the International Fluid Academy. Available from: https://healthmanagement.org/c/icu/issuearticle/thoughts-on-covid-19-fromthe-international-fluid-academy (last accessed August 03, 2021).

[14] Flinspach AN, Booke H, Zacharowski K, Balaban Ü, Herrmann E, Adam EH. High sedation needs of critically ill COVID-19 ARDS patients-a monocentric observational study. PLoS One 2021;16;e0253778.

[15] Freeman BS, Berger JS. Anesthesiology core review: part one basic exam. Chapter 92: Intravenous Fluid Therapy. Washington, DC: McGraw-Hill Education; 2014. Available from: https://accessanesthesiology.mhmedical.com/book.aspx? bookid=974.

[16] Perner A, Prowle J, Joannidis M, Young P, Hjortrup PB, Pettilä V. Fluid management in acute kidney injury. Intensive Care Med 2017;43;807-15. 